\title{
Discontinuity Preserving Noise Removal Method based on Anisotropic Diffusion for Band Pass Signals
}

\author{
Sasan Mahmoodi \\ School of Electronic and Computer Science, \\ University of Southampton \\ Southampton, $U K$ \\ sm3@ecs.soton.ac.uk
}

\begin{abstract}
A nonlinear discontinuity-preserving method for noise removal for band pass signals such as signals modulated with Binary Phase-Shift Keying (BPSK) modulation is proposed in this paper. This method is inspired by the anisotropic diffusion algorithm to remove noise and preserve discontinuities in band pass signals modulated with a single frequency. It is demonstrated here that nonlinear noise removal method for a real valued band pass signal requires a solution for a nonlinear partial differential equation which is of fourth order in space and second order in time. The results presented in this work show better performance in nonlinear noise removal for real valued band pass signals in comparison with the previous work in the literature
\end{abstract}

Keywords-Anisotropic Diffusion; Band Pass Signals; Noise Removal; Carrier Signal; Discontinuity Preservation;

\section{INTRODUCTION}

Anisotropic diffusion for edge-preserving noise removal in images is initially put forward by Perona and Malik (PM) in their seminal work [1]. Gerig et al. generalize the nonlinear noise removal method of PM to 3D volumetric MRI images [2]. A colour anisotropic diffusion is also developed by Sapiro et al. [3] to remove the noise and preserve edges in colour images. A robust estimation method to extract a piecewise smooth image from an original noisy image is suggested by Black et al. [4]. Curvature-preserving partial differential equations (PDEs) are employed by Tshumperie [5] to propose a fast anisotropic smoothing algorithm for the noise removal of multi-valued images. Luo et. al [6] propose a technique to smooth noisy images by exploiting an edge-strength strategy to improve the preservation of the details of the smoothed image. Finally a nonlinear filtering scheme based on a linear partial differential equation of the type of the Heat equation is proposed by Mahmoodi [7] to remove noise in band pass signals by preserving discontinuities and modulating carrier signal. The method proposed in [7] however can only be used for complex valued signals. In order for this method to be used for real valued signals, Hilbert transform is employed in [7] to produce complex valued signals out of their real valued ones. A new partial differential equation is therefore required for noise removal in real valued signals. This is not a trivial

MMSP'13, Sept. 30-Oct. 2, 2013, Pula (Sardinia), Italy. 978-1-4799-0125-8/13/\$31.00 (C2013 IEEE. problem. Our contribution in this paper is that a higher order partial differential equation based on the one investigated in [7] is proposed to perform the noise removal of real valued band pass signals with no requirement to use Hilbert transform to produce the imaginary part of a real valued signal. This paper is structured as follows. The theory is presented in section II. Section III deals with implementation issues. Numerical results are then presented in section IV. Conclusions are finally drawn in section $\mathrm{V}$.

\section{THEORY}

The noise removal method proposed by Mahmoodi [7] for band pass signals by preserving discontinuities is based on a linear partial differential equation of the following form:

$$
\frac{\partial u}{\partial t}=K\left(\frac{\partial}{\partial x}-j \omega_{0}\right)^{2} u
$$

with initial condition

$$
u(x, 0)=y(x)
$$

where $u: R^{2} \rightarrow C, y: R \rightarrow C, K>0 \quad$ and $\omega_{0}$ are the smoothed signal, original noisy signal, constant values respectively and $j=\sqrt{-1}$. In equation (1), it is assumed that the carrier signal has $\omega_{0}$ frequency and $u$ and $y$ are complex valued signals. Equation (1) is associated with one carrier signal with frequency $\omega_{0}$. The nonlinear noise removal proposed in [7] can therefore be only used for complex valued signals having real and imaginary parts. Signals in real life however are real valued. Such a signal requires a real valued carrier signal. The spectrum of a real valued carrier signal therefore consists of two frequency components in frequency domain in frequencies $\omega_{0}$ and $-\omega_{0}$. The anisotropic diffusion equation associated with frequency $-\omega_{0}$ is written as:

$$
\frac{\partial u}{\partial t}=K\left(\frac{\partial}{\partial x}+j \omega_{0}\right)^{2} u
$$

An initial condition similar to (2) is also used for this equation. Equations (1) and (3) can be rewritten as:

$$
\left(\frac{\partial}{\partial t}-K\left(\frac{\partial}{\partial x}-j \omega_{0}\right)^{2}\right) u=0
$$




$$
\left(\frac{\partial}{\partial t}-K\left(\frac{\partial}{\partial x}+j \omega_{0}\right)^{2}\right) u=0
$$

To design a noise removal technique for real valued signals, equations (4) and (5) need to be combined. In this paper, we propose the following equation combining equations (4) and (5):

$$
\left(\frac{\partial}{\partial t}-K\left(\frac{\partial}{\partial x}-j \omega_{0}\right)^{2}\right)\left(\frac{\partial}{\partial t}-K\left(\frac{\partial}{\partial x}+j \omega_{0}\right)^{2}\right) u=0
$$

Or

$$
\frac{\partial^{2} u}{\partial t^{2}}=2 K \frac{\partial}{\partial t}\left(\frac{\partial^{2}}{\partial x^{2}}-\omega_{0}^{2}\right) u-K^{2}\left(\frac{\partial^{2}}{\partial x^{2}}+\omega_{0}^{2}\right)^{2} u
$$

We assume the following initial conditions for equation (6):

$$
\begin{aligned}
& u(x, 0)=y(x) \\
& \frac{\partial u(x, 0)}{\partial t}=0
\end{aligned}
$$

It is noted that in equation (6), $u: R^{2} \rightarrow R, y: R \rightarrow R$ are real valued signals. By taking Laplace transform with respect to time $t$ and Fourier transform with respect to space $x$ from both sides of equation (6), it becomes clear that the resulting transfer function contains two central frequencies $\omega_{0}$ and $-\omega_{0}$. This is indeed similar to "factoring" method in the solution of wave partial differential equation, where wave equation is factored into two multiplying terms: one responsible for the wave traveling in the left direction and the other one in charge of the wave traveling in the right direction [11]. It is also important to notice that addition and/or subtraction of equations (4) and (5) does not produce the desired results and therefore it does not lead to a system capable of dealing with a real valued signal containing two carrier frequencies at $\omega_{0}$ and $-\omega_{0}$. Before we use equation (6) to propose a nonlinear filtering algorithm to remove noise from real valued band pass signals and preserve discontinuities and the carrier signal, let us investigate linear equation (6). The propagator of equation (6) is a real valued Gabor-like filter shown in figure (1) for constant values of $K=1, \omega_{0}=0.25$ and $t=1250$.

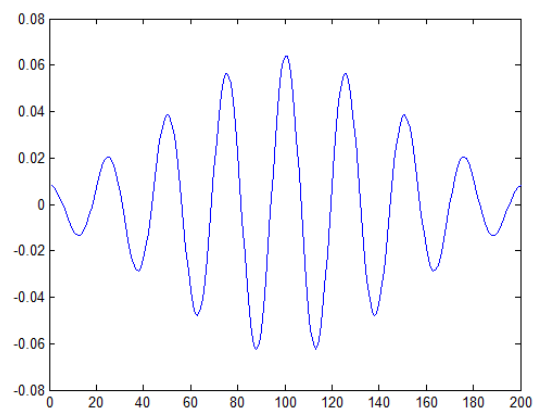

Figure 1: The propagator of equation (6)
Zero mean Gaussian noise is added to the noiseless signal shown in figure (2-a) to produce a noisy signal depicted in figure (2-b) with $\mathrm{SNR}=0.2154$. Equation (6) is applied to the noisy signal of figure (2-b) with $K=1, \omega_{0}=0.25$ and $t=1250$ to produce the smoothed signal shown in figure (2-c). The heat equation (with $K=1$ and $t=1250$ ) whose propagator is a Gaussian filter has also been applied to the noisy signal of figure (2-b) to obtain the smoothed signal shown in figure (2d). As shown in this figure, the carrier signal has been filtered out and the smoothed signal is completely distorted by the heat equation. In the next section, equation (6) with initial conditions (7) and (8) are discretized and used nonlinearly to remove the noise, and preserve discontinuities and the real valued carrier signal with frequency $\omega_{0}$.

\section{IMPLEMENTATION ISSUES}

We employ an explicit finite difference scheme for the discretization of equation (6). A central finite difference approach is used for spatial discretization and a forward finite difference scheme is employed for temporal discretization.

For the discretization purposes, equation (6) can be written in the following form:

$$
\frac{\partial^{2} u}{\partial t^{2}}=2 K \frac{\partial}{\partial t}\left(\frac{\partial^{2}}{\partial x^{2}}-\omega_{0}^{2}\right) u-\left(\frac{\partial^{2}}{\partial x^{2}}+\omega_{0}^{2}\right) K^{2}\left(\frac{\partial^{2}}{\partial x^{2}}+\omega_{0}^{2}\right) u
$$

By using an explicit discretization scheme, equation (9) can therefore be discretized as follows:

$$
\begin{aligned}
& u(x, t+\Delta t)=2 u(x, t)-u(x, t-\Delta t) \\
& +2 \Delta t K_{C}\left(E_{\omega_{0}}(u(x, t))-E_{\omega_{0}}(u(x, t-\Delta t))\right. \\
& -\Delta t^{2}\left(K _ { R } D _ { \omega _ { 0 } } \left(u(x+\Delta x, t)-2 K_{C} D_{\omega_{0}}(u(x, t))+K_{L} D_{\omega_{0}}(u(x-\Delta x, t))\right.\right. \\
& +\omega_{0}^{2} K_{C} D_{\omega_{0}}(u(x, t))
\end{aligned}
$$

where

$$
\begin{aligned}
& D_{\omega_{0}}(u(x, t))=\frac{u(x+\Delta x, t)-2 u(x, t)+u(x-\Delta x, t)}{\Delta x^{2}}+\omega_{0}^{2} u(x, t) \\
& E_{\omega_{0}}(u(x, t))=\frac{u(x+\Delta x, t)-2 u(x, t)+u(x-\Delta x, t)}{\Delta x^{2}}-\omega_{0}^{2} u(x, t) \\
& K_{C}=g\left(\left|\frac{u(x+\Delta x, t)-2 u(x, t)+u(x-\Delta x, t)}{\Delta x^{2}}+\omega_{0}^{2} u(x, t)\right|\right) \\
& K_{R}=g\left(\left|\frac{u(x+2 \Delta x, t)-2 u(x+\Delta x, t)+u(x, t)}{\Delta x^{2}}+\omega_{0}^{2} u(x+\Delta x, t)\right|\right) \\
& K_{L}=g\left(\left|\frac{u(x, t)-2 u(x-\Delta x, t)+u(x-2 \Delta x, t)}{\Delta x^{2}}+\omega_{0}^{2} u(x-\Delta x, t)\right|\right)
\end{aligned}
$$

Function $g(y)$ can be chosen as one of the following functions:

$g(y)=\exp \left(-\frac{y^{2}}{q^{2}}\right)$ 
Or

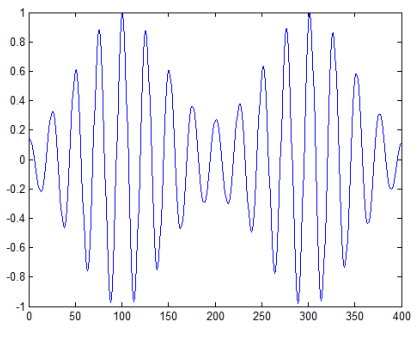

(a)

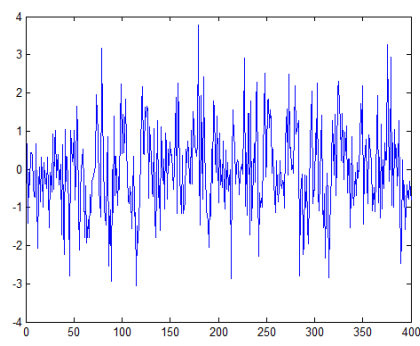

(b)

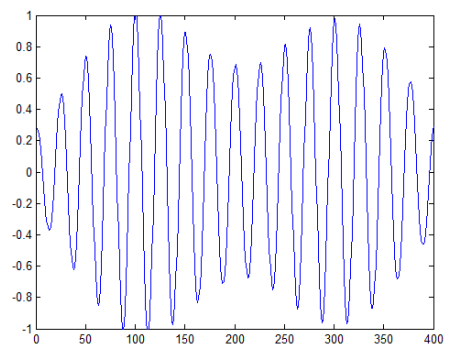

(c)

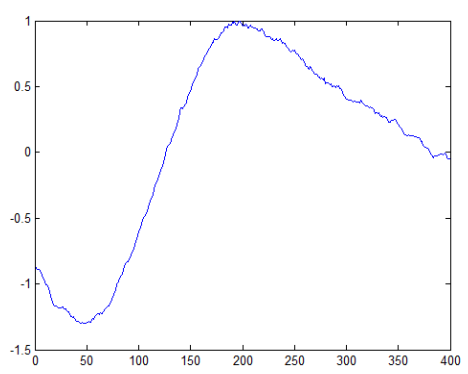

(d)

Figure 2: Linear filtering of equation (6), a) a noiseless signal with a carrier frequency of $\omega_{0}=0.25$ b) Noisy signal produced by contaminating the noiseless signal of figure (2-a) with a zero mean Gaussian noise with $\mathrm{SNR}=0.2154$, c) the signal smoothed by applying equation (6) with $K=1, \omega_{0}=0.25$ and $t=1250$ to the noisy signal of figure (2-b) d) the signal smoothed by applying the heat equation with $K=1$, and $t=1250$ to the noisy signal of figure (2-b)

$$
g(y)=\frac{1}{1+\frac{y^{2}}{q^{2}}}
$$

where $q$ is a parameter determined by users. Any discontinuity in signal components with frequencies $\omega_{0}$ and $-\omega_{0}$ would produce a local maxima in terms such as $\left(\frac{\partial^{2}}{\partial x^{2}}+\omega_{0}^{2}\right) u$ and $\left(\frac{\partial^{2}}{\partial x^{2}}-\omega_{0}^{2}\right) u$ in equation (9).

The terms $K_{R}, K_{L}$ and $K_{C}$ therefore approach to zero to preserve the discontinuity, in case a local maximum is detected in $\left(\frac{\partial^{2}}{\partial x^{2}}+\omega_{0}^{2}\right) u$ and $\left(\frac{\partial^{2}}{\partial x^{2}}-\omega_{0}^{2}\right) u$.

Throughout this paper, $\Delta x$ is set to unity. Time $t$ is treated virtually and corresponds to iterations in the algorithm. The Euclidian distance between two consecutive smoothed signals is used as a stopping criterion. If this distance is less than a threshold, the algorithm stops and the algorithm is said to have converged. Parameter $\Delta t$ is used to adjust the speed of the convergence. Large values of $\Delta t$ may increase the speed of the convergence and may also lead to the instability of the algorithm as it is expected in an explicit finite difference strategy. Lower values for $\Delta t$, on the other hand, guarantees the algorithm's convergence in the expense of lower speeds of the convergence. Finally parameter $q$ should be selected according to the amount of discontinuities in the original noiseless signals. Over-smoothing in the smoothed signal may occur if larger values for $q$ are chosen by the user. This oversmoothing is demonstrated in the smoothed signal with some smoothed (not preserved) discontinuities. Smaller values for $q$, on the other hand, in a very noisy environment, may lead to the failure of noise removal and preserving some discontinuities associated with noise rather than the original noiseless signal. Generally it is best to choose lower values for $q$ in the presence of moderate noise to preserve discontinuities. We also note that, to avoid the effects associated with the uncertainty principle [8], it is always assumed that there is at least one full cycle of the carrier signal between two consecutive discontinuities.

\section{Nuemrical Results}

In this section, we apply the nonlinear band pass filter derived in equation (10) on noisy band pass signals containing discontinuities. Figure (3-a) shows a noiseless band pass signal with frequency $\omega_{0}=0.25$ containing discontinuities. This is an example of a digital signal modulated by using Binary PhaseShift Keying (BPSK) modulation [9]. Zero mean Gaussian noise is added to this noiseless signal to result in the noisy signal with SNR =12.49 shown in figure (3-b). Our nonlinear 


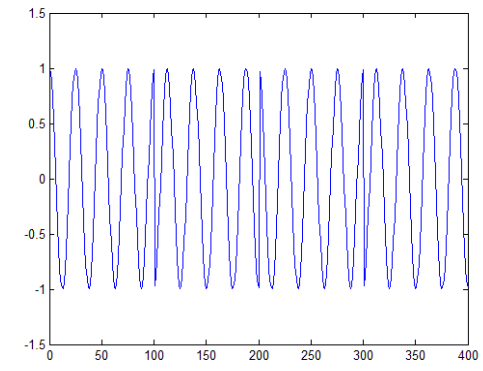

(a)

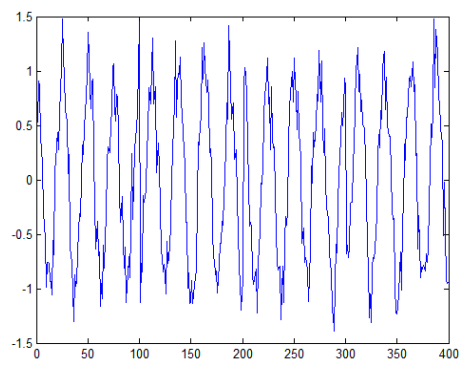

(b)

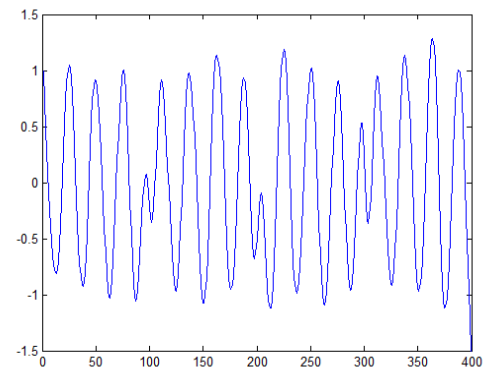

(c)

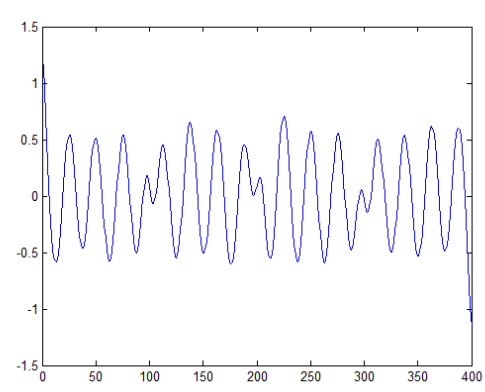

(d)

Figure 3: Noise removal in a band pass signal (BPSK modulated signal) containing discontinuities, a) Original noiseless signal with frequency $\omega_{0}=0.25$ with discontinuities at locations 100, 200 and 300, b) Noisy signal contaminated with zero mean Gaussian noise with $\mathrm{SNR}=12.49$, c) the signal smoothed with our method proposed here for $q=1$, and $\Delta t=0.005$ (d) the signal smoothed with a nonlinear low pass filter based on anisotropic equation [7] with $q=1$, and $\Delta t=0.005$

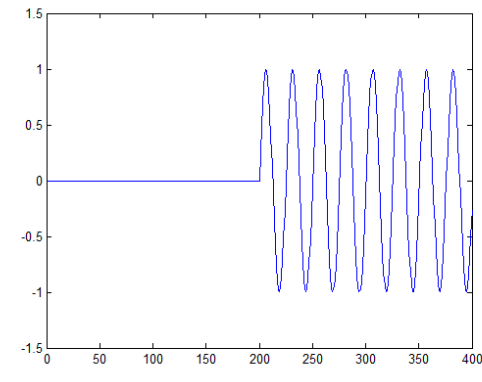

(a)

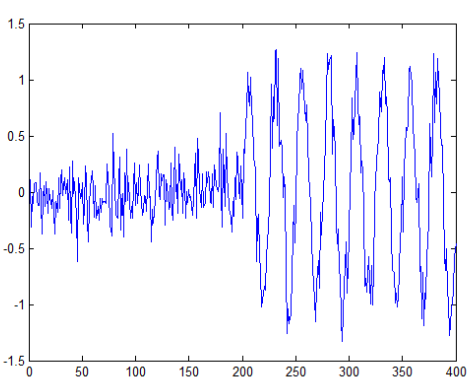

(b)

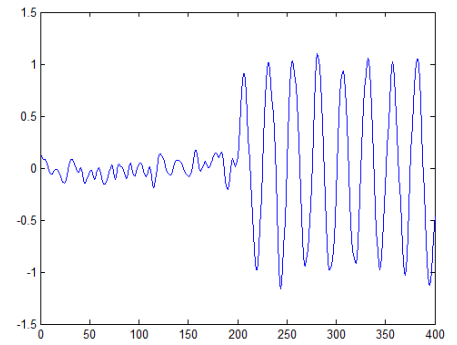

(c)

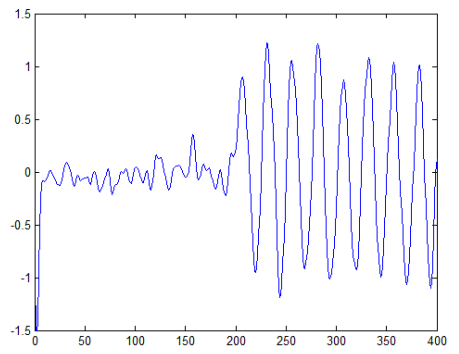

(d)

Figure (4): Noise removal of a band signal containing discontinuities a) an original noiseless band pass signal with $\omega_{0}=0.25$ containing a discontinuity at the location of $200 \mathrm{~b}$ ) Noisy signal contaminated with a zero mean Gaussian noise with the standard deviation of $0.2 \mathrm{c}$ ) The signal smoothed by the method proposed in [7] with $q=1$ and $\Delta t=0.001 \mathrm{~d}$ ) the signal smoothed by our filtering method proposed here with $q=1$ and $\Delta t=0.001$. 
filter proposed here with $q=1$ and $\Delta t=0.005$ is applied to the noisy signal of figure (3-b) to obtain the smoothed signal shown in figure (3-c). As can be seen from this figure, Noise is removed and carrier signal as well as discontinuities are preserved. It takes our nonlinear filtering algorithm only 1.26 seconds to converge to the solution shown in figure (3-c) in a 64-bit Matlab (version 7.11) running on a PC workstation with a CPU with frequency $2.67 \mathrm{GHz}$. A nonlinear low pass filter based on anisotropic diffusion equation [7] with $q=1$ and $\Delta t=0.005$ is also applied to the noisy signal of figure (3-b) to obtain the smoothed signal shown in figure (3-d). As can be seen from figure (3-d), the smoothed signal has lower amplitude owing to low pass filtering and also the discontinuities are considerably smoothed.

In the next experiment, we demonstrate that our algorithm proposed in this paper is competitive in comparison with a nonlinear band pass filter requiring to compute the imaginary part of a real signal by exploiting the Hilbert transform [7]. In the nonlinear filtering scheme proposed here however there is no need to compute the Hilbert transform of the real valued signal, i.e. our partial differential equation is applied to only real valued signals. Figure (4-a) shows a noiseless band pass signal with a discontinuity at the location of 200 and $\omega_{0}=0.25$. Zero mean Gaussian noise with a standard deviation of 0.2 is then added to the noiseless signal to produce the noisy signal of figure (4-b). The band pass nonlinear filtering process proposed in [7] with $q=1$ and $\Delta t=0.001$ is applied to the noisy signal of figure (4-b) to obtain the smoothed signal of figure (4-c). The nonlinear filtering technique proposed in this paper with $q=1$ and $\Delta t=0.001$ is also applied to the noisy signal of figure (4-b) to compute the smoothed signal shown in figure (4-d). As can be seen from figure (4), our noise removal algorithm based on a new partial differential equation proposed here, produces competitive results in comparison with the work presented in [7] with no further requirement and hassle of the Hilbert transform computation needed to compute the imaginary part of real valued signals.

\section{CONCLUSION}

A nonlinear noise removal algorithm based on a new formulation for band pass signals to preserve discontinuities is presented in this paper. The filtering algorithm proposed here demonstrates superior performance over the nonlinear low pass filter based on anisotropic diffusion. In contrast with the previous work of the noise removal for band pass signals presented in [7], the algorithm presented here has the advantage that the Hilbert transform is not required to produce complex valued signals (needed for the method proposed in [7]). Our algorithm directly is applied to the real valued signals with equivalent results obtained from the algorithm presented in [7]. Furthermore the mathematical framework presented in this paper paves the way for noise removal algorithms suitable for band pass signals containing carrier signals with more than one frequency (multi-frequency carrier signals) such as signals modulated by using Orthogonal Frequency-Division Multiplexing (OFDM) modulation scheme [10].

\section{REFERENCES}

[1] P. Perona, J. Malik, "Scale-Space and Edge Detection using Anisotropic Diffusion", IEEE Transactions on Pattern Recognition and Machine Intelligence, Vol. 12, No. 7, pp. 629-639, 1990.

[2] G. Gerig, O. Kubler, R. Kikinis, F. Jolesz, "Nonlinear Anisotropic Filtering of MRI Data" IEEE Transactions on Medical Imaging, Vol. 7, No. 11, pp. 1582-1586, 1996.

[3] G. Sapiro, D.L. Ringach, "Anisotropic Diffusion of Multi-Valued Images with Applications to Color Filtering", IEEE Transactions on Image processing, Vol. 5, No. 11, pp. 1582-1586, 1996.

[4] M.J. Black, G. Sapiro, D.H. Marimont, D. Heeger, "Robust Anisotropic Diffusion", IEEE Transactions on Image Processing, Vol. 7, No. 3, pp. 421-432, 1998.

[5] D. Tschumperie, "Fast Anisotropic Smoothing of Multi-Valued Images using Curvature-Preserving PDEs", International Journal of Computer Vision, Vol. 68, No. 1, pp. 65-82, 2006.

[6] H. Luo, L. Zhu, H. Ding, "Coupled Anisotropic Diffusion for Image Selective Smoothing”, Signal Processing, Vol. 86, No. 1, pp. 65-82, 2006.

[7] S. Mahmoodi, "Anisotropic Diffusion for Noise Removal of Band Pass Signals”, Signal Processing, Vol. 91, No.5, pp. 1298-1307, 2011.

[8] V. Harvin, B. Joricke, The Uncertainty Principle in Harmonic Analysis, Springer-Verlag, 1994.

[9] H. Stern, S. Mahmoud, Communication Systems, Pearson Prentice Hall, 2004.

[10] L. Hanzo, S.X.Nq, T. Keller, W.T. Webb, Quadrature Amplitude Modulation: From Basics to Adaptive Trellis-Coded, Turbo-Equalised and Space-Time Coded OFDM, CDMA and MC-CDMA Systems, WileyBlackwell, 2004.

[11] G.B. Arfken, H.J. Weber, Mathematical Methods for Physicists, Academic Press, the $7^{\text {th }}$ Edition, 2012. 